\title{
ChemComm
}

Check for updates

Cite this: Chem. Commun., 2019, 55,9877

Received 22nd June 2019, Accepted 22nd July 2019

DOI: $10.1039 / \mathrm{c} 9 \mathrm{cc} 04781 f$

rsc.li/chemcomm

\section{Fully independent photochemical reactivity in one molecule $\uparrow$}

\author{
David E. Marschner $\ddagger^{\mathrm{ab}}$ Christoph O. Franck, $\neq^{\mathrm{ac}}$ Doris Abt, ${ }^{\mathrm{a}}$ Hatice Mutlu (D) *ad and \\ Christopher Barner-Kowollik (D)*ab
}

\begin{abstract}
We introduce a chemically $\lambda$-orthogonal bichromophore triggered simply by different colours of light, enabling two different photochemical reactions in one molecule. Uniquely, the short wavelength $(\lambda=314 \mathrm{~nm})$ does not trigger the red-shifted reaction system $(\lambda=416 \mathrm{~nm})$, opening possibilities for the light controlled gating of specific molecular sites independent of wavelength.
\end{abstract}

Beyond fundamental scientific interest, a key quest in modern synthetic chemistry is to achieve spatiotemporal control over two (or more) different reactive moieties in a one-pot reaction mixture. ${ }^{1,2}$ Indeed, such control can be readily achieved by adopting orthogonal light-harvesting reactions. ${ }^{2}$ However, the use of such reactions is often challenging due to wavelengthdependent selectivity issues, since many reactions utilise light in the same range of wavelengths. ${ }^{3}$ For an independent reaction process, it is necessary to separate the absorption bands of the employed chromophores and therefore their respective triggers, e.g. through red-shifting. ${ }^{2}$ Only a few examples of wavelength-orthogonal ( $\lambda$-orthogonal) reaction systems have been reported so far, many of which are beset with limitations such as a specific order that needs to be followed in respect to reaction sequence. ${ }^{4}$ Often, incomplete orthogonality is observed since the visible light reactive component is also triggered when using UV light. Thus, the reaction is only orthogonal when

\footnotetext{
${ }^{a}$ Macromolecular Architectures, Institute for Chemical Technology and Polymer Chemistry, Karlsruhe Institute of Technology (KIT), Engesserstr. 18, 76131 Karlsruhe, Germany. E-mail: christopher.barner-kowollik@kit.edu

${ }^{b}$ School of Chemistry, Physics and Mechanical Engineering, Queensland University of Technology (QUT), 2 George Street, Brisbane, QLD 4000, Australia. E-mail: christopher.barnerkowollik@qut.edu.au

${ }^{c}$ Department of Chemical Engineering and Biotechnology, University of Cambridge Philippa Fawcett Drive, Cambridge, CB3 OAS, UK

${ }^{d}$ Soft Matter Synthesis Laboratory, Institute for Biological Interfaces, Karlsruhe Institute of Technology (KIT), Hermann-von-Helmholtz-Platz 1, 76344 Karlsruhe, Germany. E-mail: hatice.mutlu@kit.edu

$\dagger$ Electronic supplementary information (ESI) available: In-depth analysis including NMR, UV/Vis and GC-MS spectra providing further proof of the introduced concept. See DOI: $10.1039 / \mathrm{c} 9 \mathrm{cc} 04781 \mathrm{f}$

\# These authors contributed equally.
}

irradiation takes place starting with the longest wavelength and then proceeding to the shorter one. To overcome this sequence dependent procedure, it is possible to use reactions that are either deactivated or unreactive in the visible light range. Indeed, a pathway to complete $\lambda$-orthogonality is provided by the utilisation of reversible photochemistry, for example through the use of styrylpyrene, as it exclusively dimerises when irradiated with visible light and not under UV-A irradiation., ${ }^{5,6}$ In fact, it was recently reported that styrylpyrene can be combined with other light-harvesting groups such as anthracene to enable an additional second dimerisation reaction. ${ }^{7,8}$ An alternative chromophore that can undergo a photoreaction under UV-A light while remaining unreactive in the visible light range is $o$-methyl benzaldehyde $(o$-MBA), which generates an $o$-quinodimethane species under excitation with light that readily reacts with maleimides. ${ }^{9}$ Compared to anthracene, $o$-MBA has the advantage that it can react with various molecules featuring double bonds (e.g. maleimides, fumarates), while anthracene is able to undergo dimerisation with itself only. In combination with both a reversible as well as visible light system, this could pave a way for novel $\lambda$-orthogonal systems.

The motivation underpinning the current study is to combine several light-harvesting chromophores into one molecule that can undergo site specific cycloaddition reactions at different wavelengths in a $\lambda$-orthogonal fashion. By choosing different linkage molecules, e.g. star linkers, this idea can be expanded from a system with two functional groups to multiple reactions sites - a polychromophoric molecule. To demonstrate our concept, establish a platform technology and enable in-depth analytical characterisation, a bi-linker was selected as concept molecule. $o$-MBA was one of the light-responsive moieties of choice, which can be triggered with UV-A light to react with preferably electron-deficient double bonds, such as maleimides, yet can also self-dimerise. Nevertheless, in previous studies we evidenced that $o$-MBA preferentially reacts in an efficient manner with activated dienes, such as maleimides, than undergoing self-dimerisation..$^{10,11}$ In addition, $o$-MBA $(310-350 \mathrm{~nm})$ has shown to be suitable for sequence-dependent $\lambda$-orthogonal reactions in combination with tetrazoles $(270-310 \mathrm{~nm})$, where the higher wavelength 


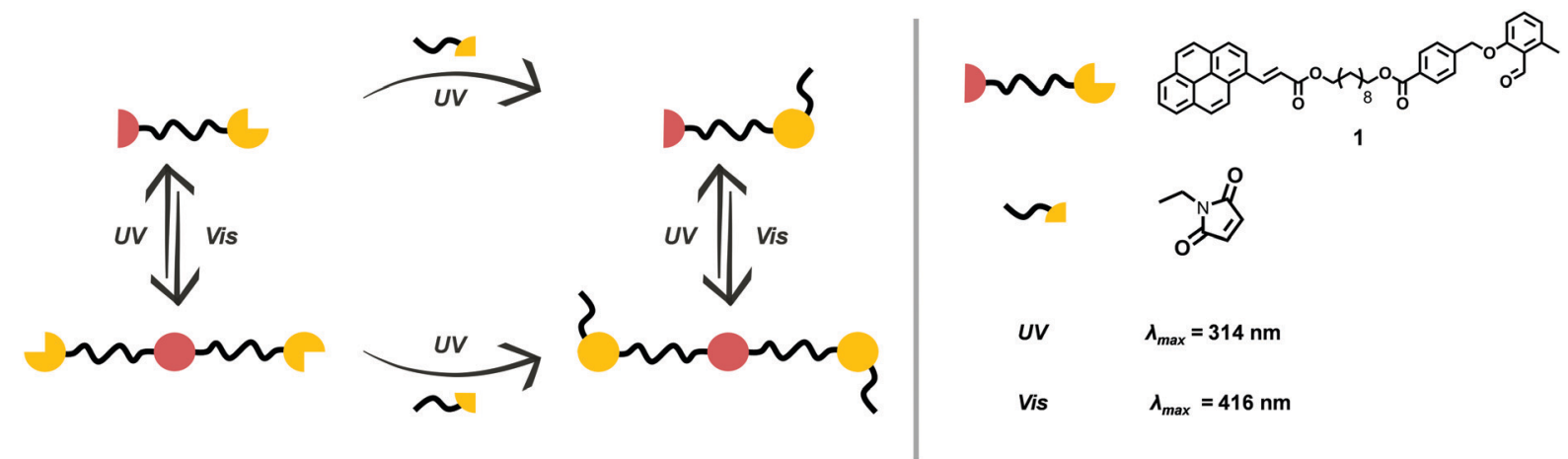

Fig. 1 Schematic overview showing the investigated bichromophore with its pyrenyl cinnamic acid (PCA) and ortho-methylbenzaldehyde (o-MBA) end groups as well as the anticipated orthogonal synthesis platform possible upon irradiation with light.

component is fully triggered first. ${ }^{10}$ Recently, we demonstrated that $o$-MBA and tetrazoles can be used in a sequence-independent $\lambda$-orthogonal fashion, however, only through control of the respective number of photons and solvents used during irradiation. ${ }^{12}$ The second chromophore is a cinnamic acid derivative, which can undergo a reversible [2+2] photo-cycloaddition. To achieve a sufficient separation of the absorption bands - and thus their triggering wavelengths - for the two light-harvesting groups, functionalisation of the cinnamic acid with a pyrene unit is preferential as it is known to red-shift double bonds that can subsequently undergo a [2+2] cycloaddition within the visible light regime (e.g. styrylpyrene, pyrene aryl tetrazole). ${ }^{6,13,14}$ With such a bichromophoric molecule, it is anticipated that two different pathways completely independent of each other may result in the same product as depicted in Fig. 1.

To determine the reactivity of the cinnamic acid double bond, a proof-of-concept molecule was synthesised. Specifically, a pyrenyl cinnamic acid (PCA) derivative (2, refer to Fig. 3) featuring a butanol chain instead of being linked to $o$-MBA. 2 was used to study the dimerisation as well as the dissociation behaviour of the double bond without the possibility of triggering the $o$-MBA moiety, especially when irradiating the molecule with UV-A light. As expected, functionalisation of cinnamic acid with pyrene introduced a red-shift of $c a .100 \mathrm{~nm}$ to $\lambda_{\max }=370 \mathrm{~nm}$ of the resulting compound when compared to cinnamic acid: $\lambda_{\max }=272 \mathrm{~nm}$, which can be explained by the extension of the conjugated system. ${ }^{6,14,15}$ Exclusively under irradiation with visible light $\left(\lambda_{\max }=416 \mathrm{~nm}\right) 2$ undergoes dimerisation, but also isomerisation, evidenced by NMR (refer to ESI, $\dagger$ Fig. S1-S3). In addition, the UV/Vis spectra indicate the formation of the targeted photoproduct (Fig. 2). When 2 undergoes dimerisation, the extended conjugation system is reduced, and the molecule converts to an unconjugated spectrum featuring two absorption bands at $\lambda=333$ and $348 \mathrm{~nm}$, indicating a successful photoreaction. ${ }^{16}$ According to absorbance measurements, maximum conversion (79\% photoproduct, calculated from ${ }^{1} \mathrm{H}$ NMR spectra via integration of distinct resonances for the different species) was reached after $60 \mathrm{~min}$ (refer to ESI, $\dagger$ Fig. S4A).

In theory, $[2+2]$ cycloadditions are reversible and can be reverted to their starting materials once they are irradiated with a wavelength that is shorter than the one required for the respective

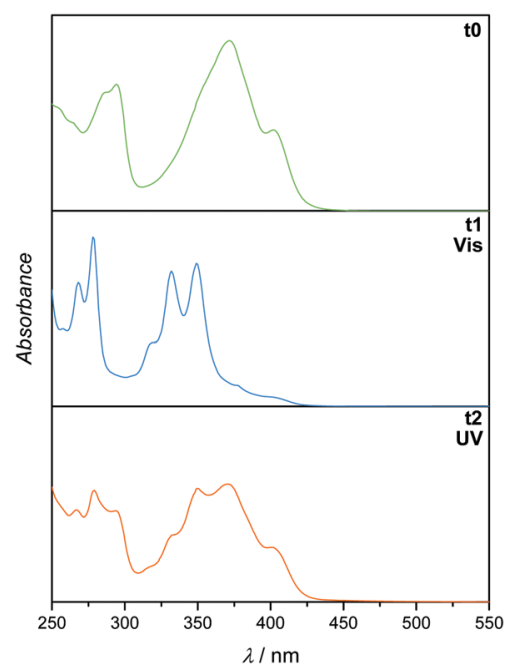

Fig. 2 UV/Vis spectra (recorded in DCM) of 2 before irradiation (t0), after irradiation with visible light (t1) and subsequent irradiation with UV light (t2).

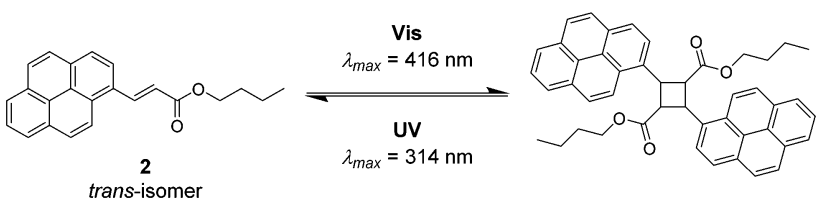

Fig. 3 Reversible [2+2] cycloaddition of pyrenyl cinnamic acid 2 under irradiation with visible $\left(\lambda_{\max }=416 \mathrm{~nm}\right)$ and irradiation with UV-A light $\left(\lambda_{\max }=314 \mathrm{~nm}\right)$. Irradiation with UV-A light introduces not only the cycloreversion, yet also the isomerisation of $\mathbf{2}$.

dimerisation reaction. ${ }^{3}$ In order to investigate the cycloreversion, the 2-dimer was irradiated with UV-A light $\left(\lambda_{\max }=314 \mathrm{~nm}\right)$. Consequently, the reaction mixtures were characterised in-depth by utilising NMR spectroscopy which showed almost complete reversion of the dimer (3\% remaining dimer, calculated from ${ }^{1} \mathrm{H}$ NMR spectra) to the starting trans-isomer of 2 that partly underwent photoisomerisation to its cis-structure (ESI, $\dagger$ Fig. S1 and S2). In a similar fashion to the dimerisation reaction, dissociation was observed after $60 \mathrm{~min}$ (according to the absorbance measurements, refer to the ESI, $\dagger$ Fig. S4B). The remaining traces of dimeric structures can also be observed in the UV/Vis 
spectra since the trace from $\mathrm{t} 1$ did not revert to $\mathrm{t} 0$, but rather displays a composite of the two traces. However, because the absorbance spectra of all compounds (trans- and cis-2, as well as the photoproducts) are overlapping, they cannot be used to determine the exact conversion. To ensure that the molecules are not affected during irradiation, the samples were analysed via GC-MS (ESI, $\dagger$ Fig. S5-S7), though no fragments with smaller mass values could be detected confirming that no photodamage took place.

Next, the $o$-MBA functionality in bichromophore $\mathbf{1}$ was investigated. Irradiation of 1 with UV-A light $\left(\lambda_{\max }=314 \mathrm{~nm}\right)$ should introduce a [4+2] cycloaddition of $o$-MBA with $\mathrm{N}$-ethyl maleimide (Et-Mal). Indeed, subsequent analysis via NMR spectroscopy showed complete consumption of both $o$-MBA's aldehyde and methyl resonances (refer to ESI, $\dagger$ Fig. S7).

With the irreversible ligation site of $\mathbf{1}$ fully functional, the visible light triggered reversible PCA was subsequently investigated. When 1 was irradiated with $\lambda_{\max }=416 \mathrm{~nm}$, the double bond dimerised forming a cyclobutane structure that was reversible under UV-A irradiation $\left(\lambda_{\max }=314 \mathrm{~nm}\right)$. The reaction was analysed via ${ }^{1} \mathrm{H}$ NMR (ESI, $\dagger$ Fig. S10) as well as UV/Vis spectroscopy (ESI, $\dagger$ Fig. S9) and the results were completely analogous to the small molecule study of 2. Comparable to the test molecule 2, ${ }^{1} \mathrm{H}$ NMR showed new magnetic resonances for the dimerisation reaction that almost completely disappeared during dissociation. UV/Vis spectroscopy depicted the decrease of the broad absorption band representing the fully conjugated system $\left(\lambda_{\max }=371 \mathrm{~nm}\right)$ of the trans-isomer. Subsequent irradiation with UV-A light did not convert the spectrum back to its starting pattern but a mixture of monomeric and dimeric structures, which is similar to that of the small molecule study (2).

Having successfully demonstrated both the irreversible [4+2] cycloaddition of $o$-MBA and the reversible [2+2] cycloaddition of PCA along with their independence from each other, the complete synthesis platform with subsequent orthogonal and selective absorption was carried out (refer to Fig. 1). As expected from the small molecule study, the reaction system can be irradiated with UV light triggering the $o$-MBA unit to undergo a reaction with Et-Mal. ${ }^{1} \mathrm{H}$ NMR clearly shows the consumption of the $o$-MBA (refer to ESI, $\dagger$ Fig. S10). However, the double bond underwent isomerisation from the trans- to the cis-structure, alongside small amounts of dimerisation, which can be detected via NMR measurements, and small amounts of the previously discussed absorption pattern during UV/Vis spectroscopy (Fig. 4). A plausible reason for the traces of dimer molecules is the broad emission spectrum of the utilised UV lamps that also feature small emissions up to wavelengths of approximately $400 \mathrm{~nm}$ (refer to the ESI, $\dagger$ Fig. S24). Subsequent irradiation of the same mixture with visible light $\left(\lambda_{\max }=416 \mathrm{~nm}\right)$ introduced dimerisation of the double bond (Fig. 4), successfully proving the reaction pathway utilising UV and succeeding visible light irradiation of the reaction system shown in Fig. 1 .

To explore the complementary route (depicted in Fig. 1), the sequence of irradiation was reversed by first treating the starting material 1 with visible light. The result indicated the successful dimerisation of the double bond, while both the aldehyde and methyl functionalities of $o$-MBA remained intact. Subsequent

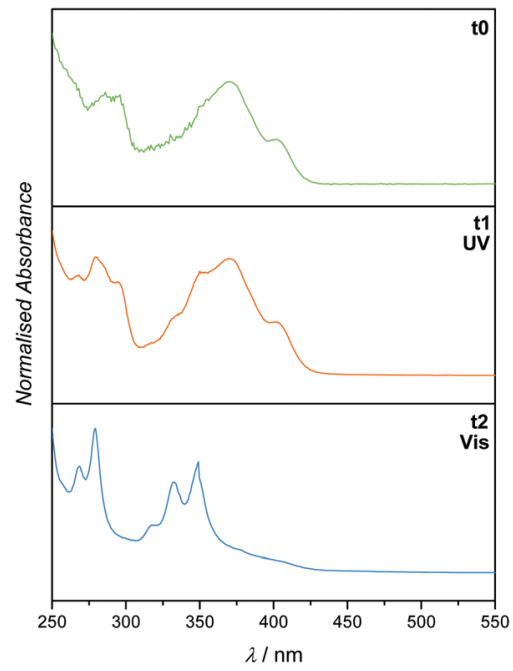

Fig. 4 UV/Vis spectra (recorded in DCM) of 1 before irradiation (t0), after irradiation with UV light (t1) and subsequent irradiation with visible light (t2). Irradiation took place in the presence of Et-Mal.

irradiation with UV light, however, induced not only the irreversible cyclisation reaction of $o$-MBA, but also the dissociation of the cyclised product generated from the previous step due to the reversible nature of the pyrenyl cinnamic acid system (refer to Fig. 5). Isomerisation of the double bonds also occurred during the UV treatment. Therefore, the complete wavelength-orthogonality only applied to the first step, where the uniquely designed compound 1 was involved. Although the UV treatment in the second step could not trigger the reaction selectively, it produced the same intermediate product as the previous route, and the re-exposure of it to visible light can again trigger the dimerisation reaction, and generate the desired final product (refer to Fig. 6).

All described molecules proved to be bench stable for over six months at ambient conditions, suggesting a high resistance

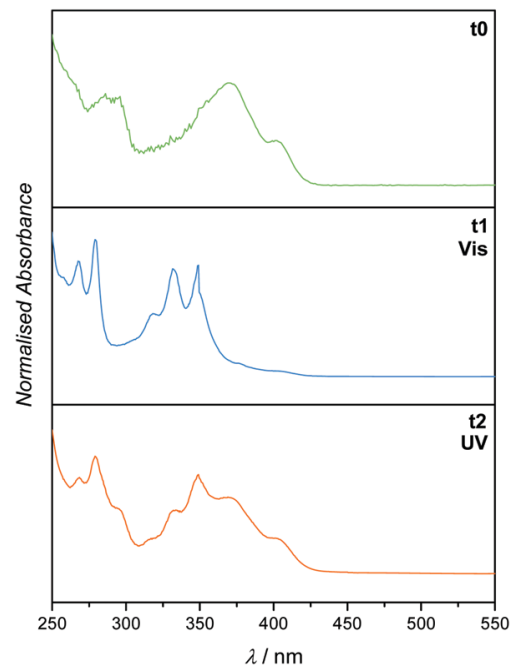

Fig. 5 UV/Vis spectra (recorded in DCM) of 1 before irradiation (t0), after irradiation with visible light (t1) and subsequent irradiation with UV light (t2). Irradiation took place in the presence of Et-Mal. 


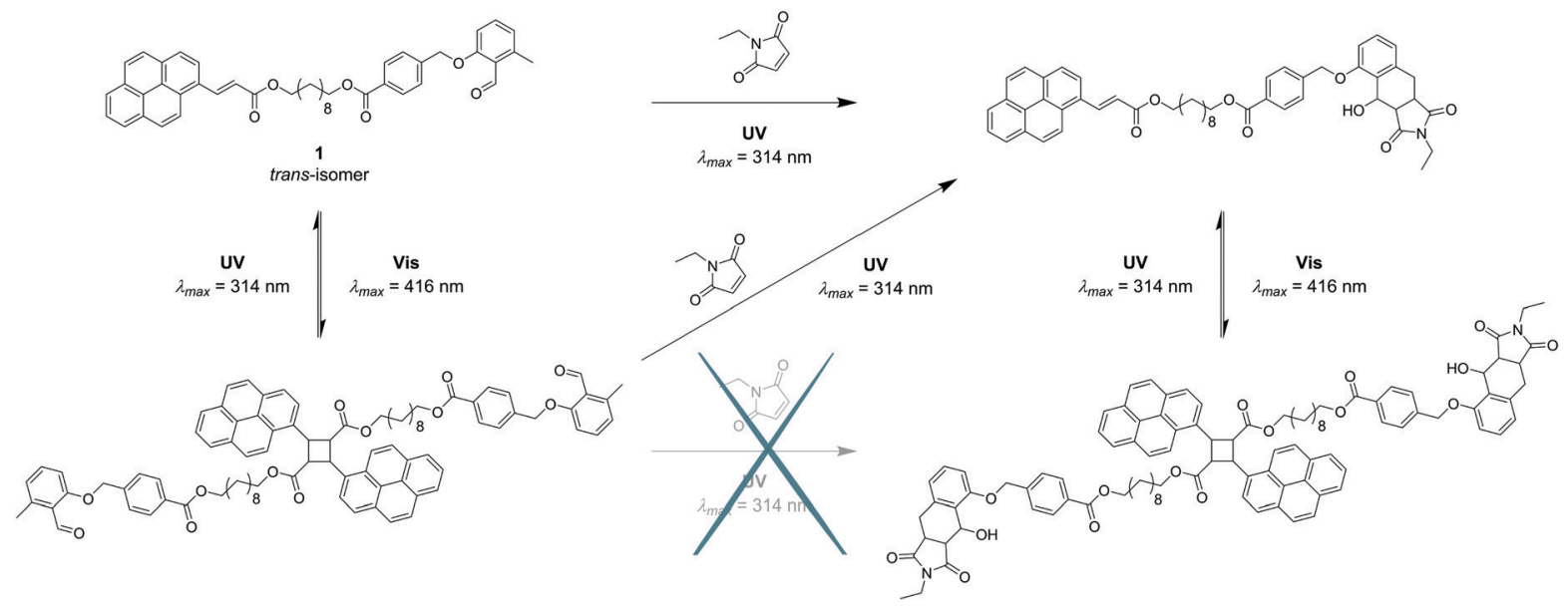

Fig. 6 Reaction scheme of 1 showing the anticipated orthogonal synthesis platform under irradiation with light as well as the pathways that were established via analysis with NMR and UV/Vis spectroscopy. The bichromophore 1 can undergo a first $\lambda$-orthogonal reaction by irradiation with either visible or UV light, while the subsequent inversion of the irradiation sequence results in the same final product.

against degradation, non-triggered cycloaddition or unwanted side reactions. In addition, initial tests that replaced Et-Mal with a polyethylene glycol featuring maleimide as an end group showed promising results (refer to ESI, $\dagger$ Chapter S1.2), suggesting that this system could also be applicable in polymer chemistry and materials science, which will be explored in future research.

In summary, we introduce a light-harvesting system in which two functional units are orthogonal to each other within the same molecule. These groups can undergo two different, side-specific photoreactions independently of each other within one molecule, exclusively by the choice of different wavelengths (UV-A and visible light). It was observed that in subsequent steps alternating UV and visible light irradiation, the same end product can be obtained. The molecule features highly interesting properties including bench stability, which enables a high sample portability. In future research, the photoreactive groups may be exchanged allowing the system to be finetuned to different applications or to introduce subsequent $\lambda$-orthogonal reaction steps. In addition, the bi-linker could be replaced with a linker featuring multiple light-harvesting groups for the synthesis of a probe that can be used as a portable and stable wavelength marker for various light regimes. The expansion of the shown concept from bi- to polychromophores opens exciting possibilities not only as wavelength markers, yet also as dynamic and reprogrammable materials. Especially through incorporation of more than one photo reversible light-harvesting group, such a polychromophore linker could be used to tailor materials to different applications and further reprogramming for specific needs. By irradiating these functional materials with different wavelengths, another part of the linker is triggered to further crosslink or - in the case of reversible photoreactions - de-crosslink the materials making them stiffer, softer or changing other physical properties such as colour. Most importantly, all these applications are combined in a completely $\lambda$-orthogonal single molecule that also features a long lifetime without any degradation processes taking place.

C. B.-K. acknowledges funding from the Karlsruhe Institute of Technology (KIT), the Australian Research Council (ARC) via a Laureate Fellowship underpinning his photochemical research program and the German Research Council (DFG). C. B.-K. acknowledges additional support under Germany's Excellence Strategy via the Excellence Cluster 3D Matter Made to Order (EXC-2082-390761711).

\section{Conflicts of interest}

There are no conflicts to declare.

\section{Notes and references}

1 A. Blanc and C. G. Bochet, J. Org. Chem., 2002, 67, 5567-5577.

2 H. Frisch, D. E. Marschner, A. S. Goldmann and C. Barner-Kowollik, Angew. Chem., Int. Ed., 2018, 57, 2036-2045.

3 G. Kaur, P. Johnston and K. Saito, Polym. Chem., 2014, 5, 2171-2186.

4 M. J. Hansen, W. A. Velema, M. M. Lerch, W. Szymanski and B. L. Feringa, Chem. Soc. Rev., 2015, 44, 3358-3377.

5 V. X. Truong, F. Li, F. Ercole and J. S. Forsythe, ACS Macro Lett., 2018, 7, 464-469.

6 D. E. Marschner, H. Frisch, J. T. Offenloch, B. T. Tuten, C. R. Becer, A. Walther, A. S. Goldmann, P. Tzvetkova and C. Barner-Kowollik, Macromolecules, 2018, 51, 3802-3807.

7 H. Frisch, F. R. Bloesser and C. Barner-Kowollik, Angew. Chem., Int. Ed., 2019, 58, 3604-3609.

8 S. Bialas, L. Michalek, D. E. Marschner, T. Krappitz, M. Wegener, J. Blinco, E. Blasco, H. Frisch and C. Barner-Kowollik, Adv. Mater., 2019, 1807288.

9 T. Gruendling, K. K. Oehlenschlaeger, E. Frick, M. Glassner, C. Schmid and C. Barner-Kowollik, Macromol. Rapid Commun., 2011, 32, 807-812.

10 K. Hiltebrandt, M. Kaupp, E. Molle, J. P. Menzel, J. P. Blinco and C. Barner-Kowollik, Chem. Commun., 2016, 52, 9426-9429.

11 T. Gegenhuber, L. De Keer, A. S. Goldmann, P. H. M. Van Steenberge, J. O. Mueller, O. Reyniers, J. P. Menzel, D. R. D'hooge and C. Barner-Kowollik, Macromolecules, 2017, 50, 6451-6467.

12 J. P. Menzel, F. Feist, B. Tuten, T. Weil, J. P. Blinco and C. BarnerKowollik, Angew. Chem., Int. Ed., 2019, 58, 7470-7474.

13 N. P. Kovalenko, A. T. Abdukadyrov, V. I. Gerko and M. V. Alfimov, J. Photochem., 1980, 12, 59-65.

14 P. Lederhose, K. N. R. Wüst, C. Barner-Kowollik and J. P. Blinco, Chem. Commun., 2016, 52, 5928-5931.

15 E. Havinga and R. J. F. Nivard, Recl. Trav. Chim. Pays-Bas, 1948, 67, 846-854.

16 T. Doi, H. Kawai, K. Murayama, H. Kashida and H. Asanuma, Chem. - Eur. J., 2016, 22, 10533-10538. 